\title{
A New Therapy for Human Endometriosis: The Therapeutic Value of Leukotriene Receptor Antagonist for Endometriosis
}

\author{
Masao Sugamata $^{*}$, Tomomi Ihara ${ }^{1}$, Ichiro Uchiide ${ }^{2}$ \\ ${ }^{1}$ Department of Pathology, Tochigi Institute of Clinical Pathology, Tochigi, Japan \\ ${ }^{2}$ Department of Obstetrics and Gynecology, Japan Red Cross Hospital Omori, Tokyo, Japan \\ Email: ${ }^{*}$ mspathol@beige.ocn.ne.jp
}

Received 21 April 2015; accepted 7 June 2015; published 10 June 2015

Copyright @ 2015 by authors and Scientific Research Publishing Inc.

This work is licensed under the Creative Commons Attribution International License (CC BY). http://creativecommons.org/licenses/by/4.0/

c) (7) Open Access

\begin{abstract}
Some drugs that regulate estrogen are currently used as therapy for endometriosis. However, these medical treatments have significant side effects and patients who hope to become pregnant cannot overcome infertility. To compare morphological alterations and clinical symptoms, revised American Fertility Society (r-AFS) scores were compared between patients with and without leukotriene receptor antagonist (LTR-A) treatment. LTR-A-treated cases showed significantly decreased r-AFS score. Furthermore, a significant correlation was seen between r-AFS score and LTR-A treatment period. In treated cases, clinical symptoms decreased and some patients achieved pregnancy. Morphologically, lesions in LTR-A-treated cases showed apoptotic fibroblasts and degeneration of collagen fibers. Our findings revealed that LTR-As had significant therapeutic value for the treatment of human endometriosis. Anti-LT therapy appears efficacious not only in the treatment of clinical symptoms and lesions, but also in improving fertility.
\end{abstract}

\section{Keywords}

Endometriosis, Leukotriene Receptor, Apoptosis, r-AFS Score, Infertility

\section{Introduction}

Endometriosis is a disease characterized by the ectopic presence of endometrial tissue. Patients with the condition show severe adhesions on the pelvic organs and peritoneal surfaces, and clinically they experience dysmenorrhea. The progression of endometriosis is linked to estrogen [1], so drugs that regulate estrogen are in current

${ }^{*}$ Corresponding author.

How to cite this paper: Sugamata, M., Ihara, T. and Uchiide, I. (2015) A New Therapy for Human Endometriosis: The Therapeutic Value of Leukotriene Receptor Antagonist for Endometriosis. Open Journal of Obstetrics and Gynecology, 5, 313-318. http://dx.doi.org/10.4236/ojog.2015.56045 
use for endometriosis patients. However, these pharmacotherapies have significant side effects, and patients often discontinue therapy. Inasmuch as endometriosis is closely related to infertility [2], development of more tolerable therapies for infertility patients with this condition is imperative.

We have recently found that hypersensitivity reactions involving the activation and degranulation of infiltrating numerous mast cells are observed in the lesions of endometriosis patients [3]. Furthermore, we have demonstrated in an experimental rat model of endometriosis that similar hypersensitivity reactions occur in lesion areas [4]. Moreover, a leukotriene receptor antagonist (LTR-A), as a form of anti-allergic drug, offered significant therapeutic benefits in the rat model [5]. The present study evaluated the therapeutic effects of LTR-As on human endometriosis.

\section{Materials and Methods}

\subsection{Patients and Anti-Leukotriene Therapy}

A total of 25 patients with endometriosis (including 10 patients with infertility; mean age, $32.7 \pm 7.80$ years; mean CA125 titer, $44.42 \pm 21.07 \mathrm{U} / \mathrm{ml}$ were prescribed a montelukast sodium (10 mg/day) as a LTR-A. Mean duration of treatment before laparoscopic surgery was 66.7 days (minimum, 14 days; maximum, 90 days). As a comparative control, 20 endometriosis patients (including 10 patients with infertility; mean age, $30.4 \pm 6.98$ years; mean titer of CA125, $53.11 \pm 17.14 \mathrm{U} / \mathrm{ml}$ ) with no pharmacotherapy were also examined and evaluated. All examinations were performed after the patients provided informed consent in accordance with the Helsinki declaration.

\subsection{Clinical Symptoms (Pain Evaluation) and Revised American Fertility Society (r-AFS) Score}

With regard to the evaluation of pain, we investigated the quantity of prescribed nonsteroidal anti-inflammatory drugs (NSAIDs) for 19 patients on LTR-A (mean duration of LTR-A treatment before laparoscopic surgery: 73.6 days; range, 56 - 90 days) who complained of dysmenorrhea and had taken NSAIDs customarily, and performed evaluations using the nonparametric one-sample sign test. As a comparative control, 20 patients with no LTR-A therapy were also examined and evaluated.

Patients were examined laparoscopically and evaluated for fibrous adhesion formation using the r-ASF score [6]. Clinical symptoms of endometriosis, such as pelvic pain (including dysmenorrheal pain) and infertility, were also evaluated. Patients with LTR-A treatment continued to take the drug postoperatively and received follow-up.

Mean r-AFS scores were calculated, and compared between cases with and without LTR-A treatment using the Mann-Whitney $U$ test. Correlations between score and LTR-A treatment period were also determined using the Spearman rank-correlation coefficient.

In all analyses, values of $p<0.05$ were considered statistically significant.

\subsection{Light and Electron Microscopic Analysis}

Tissues from endometriosis lesions in patients treated with and without LTR-A were collected via laparoscopic surgery. For light microscopy, samples were fixed with $10 \%$ buffered formalin, dehydrated in a routine manner, and embedded in paraffin. Sections 5 - $\mu$ m thick were stained with hematoxylin and eosin, and also with toluidine blue to identify mast cells [4] [5] [7]. To confirm the existence of LTRs on endometriosis, immunohistochemical staining for cysteinyl leukotriene receptor (CysLT) was performed using the streptoavidin-biotin method (Histofine SAB-PO Kit; Nichirei, Tokyo, Japan). The primary antibodies used were polyclonal antibody to CysLT1 and polyclonal antibody to CysLT2 (Acris Antibodies, San Diego, CA).

For electron microscopy, tissue samples were fixed immediately with $1 \%$ glutaraldehyde and $4 \%$ formalin for $6 \mathrm{~h}$ at $4^{\circ} \mathrm{C}$ and rinsed in 0.1-M cacodylate buffer overnight. These tissues were postfixed with $1 \%$ osmium tetraoxide and embedded in Epon 812 resin (Nissin EM, Tokyo, Japan). Ultrathin section were prepared with an ultramicrotome (Model MT-XL; RMC, Tucson, AZ), double-stained with uranyl acetate and lead citrate, and examined under an electron microscope (Model JEM1400; JEOL, Tokyo, Japan). Apoptotic appearance was evaluated based on the characteristics of ultrastructural processes in the formation of apoptotic bodies, as described previously [8]. 


\section{Results}

\subsection{Clinical Symptoms and r-AFS Score}

With regard to clinical symptoms, LTR-A treatment made the amount of taken NSAIDs for 19 patients, who complained of pelvic pain including dysmenorrhea, decrease as follows; eight of the 19 patients reported decreased ingestion of prescribed NSAIDs, and five of the 19 patients took no NSAIDs. These data suggest a degree of pain relief with LTR-A ( $p=0.0002$; nonparametric one-sample sign test). On the other hand, as for all patients with no LTR-A therapy, there was no alteration in the quantity of prescription of NSAIDs.

Moreover, 4 of 10 infertility patients on LTR-As who continued to take the drug postoperatively became pregnant within 3 - 4 months postoperatively, whereas none of the 10 infertility patients without LTR-A treatment achieved pregnancy.

LTR-A treatment cases showed significant decreases in r-AFS score when compared with controls (Figure 1(A); no treatment: $78.10 \pm 20.83$; LTR-A treatment: $40.80 \pm 23.32$; $p<0.0001$; Mann-Whitney $U$ test). Furthermore, the decrease in r-AFS score correlated significantly with the length of LTR-A treatment (Figure 1(B), $P<0.0001$; Spearman rank correlation coefficient).

In laparoscopic surgery for LTR-A-treated patients, the area of adhesion was small and fragile. Surgical lysis was therefore easier and the area damaged by lysis was smaller compared to patients with no such treatment (Figures 2(A)-(D)).

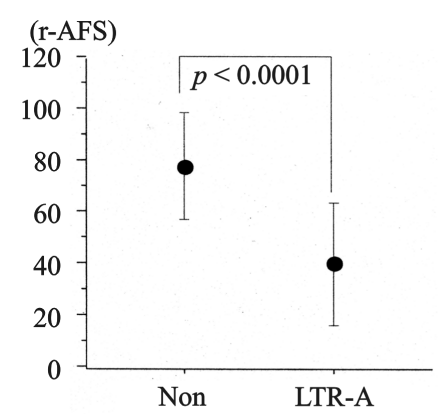

(A)

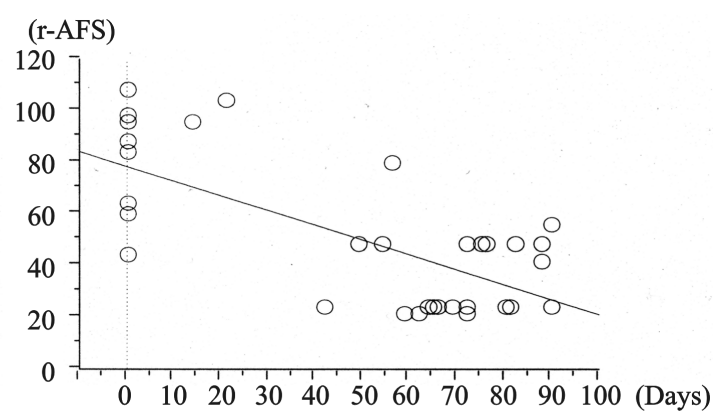

(B)

Figure 1. Evaluations of r-AFS score. (A) Comparison between no treatment and LTR-A treatment. Points and error bars indicate mean and \pm 1 SD of r-AFS score. (No treatment: $78.10 \pm 20.83$; LTR-A treatment: $40.80 \pm 23.32 ; p<0.0001$, MannWhitney $\mathrm{U}$ test). (B) Correlation of r-AFS score with LTR-A treatment period. The line indicates simple regression $(p<$ 0.0001; Spearman rank correlation coefficient).
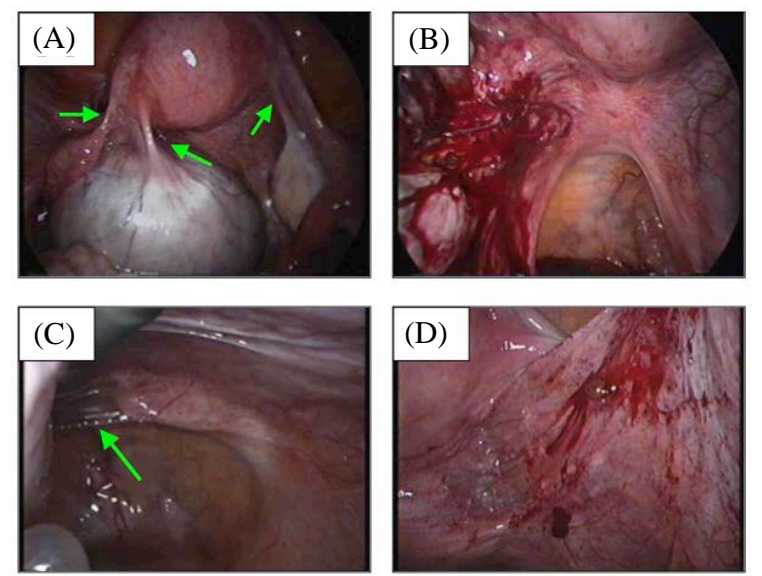

Figure 2. Comparison of laparoscopic surgery results between no treatment and LTR-A-treated patients. (A) (B) No treatment patient. The area of adhesions is large ((A): arrow), and the amount of bleeding after surgical lysis is large (B). (C) (D) LTR-A-treated patients. Compared with no-treatment patients, LTR-A-treated patients show milder adhesions behind the ovaries. The adhering tissue is very fragile and membranous ((C): arrow), and the amount bleeding after surgical lysis is small (D). 


\subsection{Light and Electron Microscopic Analysis}

In all endometriosis lesions, CysLT-positive cells were diffusely observed. Positive reactions were detected in ectopic endometrial cells, but also in fibroblasts, mast cells and endothelial cells (Figure 3(A) and Figure 3(B)).

Morphologically, in proliferative lesions of LTR-A-treated endometriosis patients, both inhibition of mast cell activation and apoptosis of numerous proliferating fibroblasts were observed in lesion areas of LTR-A-treated cases. Furthermore, collagen fibers around these apoptotic fibroblasts exhibited degenerative changes; specifically, fibers were filamentous, thin, and partially condensed (Figures 4(A)-(D)). These data indicate a decrease in extensive pelvic adhesions, and we also found that laparoscopic lysis of adhesions in LTR-A-treated patients was easier.

\section{Discussion}

In this study, our light and electron microscopic findings for endometriosis patients treated with LTR-As resembled those in LTR-A-treated experimental rat endometriosis [5]. These findings support the notion that LTR-As provide relief for patients from severe clinical symptoms and adhesive lesions.
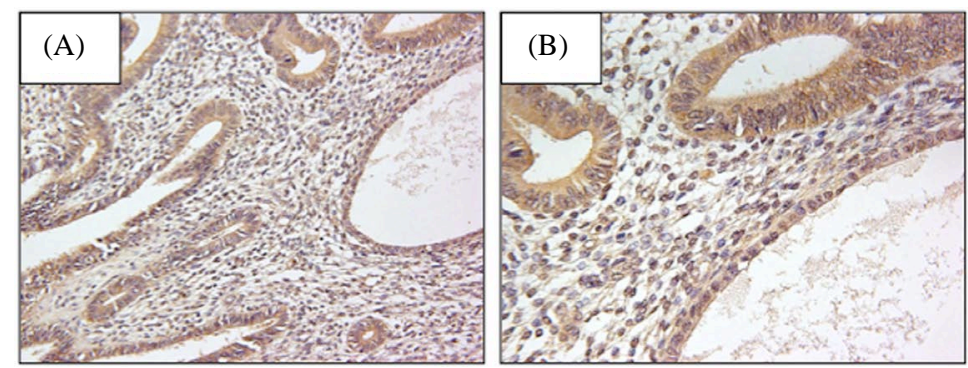

Figure 3. Detection of CysLT on endometriosis lesions by immunohistochemical staining. Brown color shows a positive reaction, as detected in ectopic endometrial cells, fibroblasts, mast cells and endothelial cells. (A) $\times 200$; (B) $\times 400$.
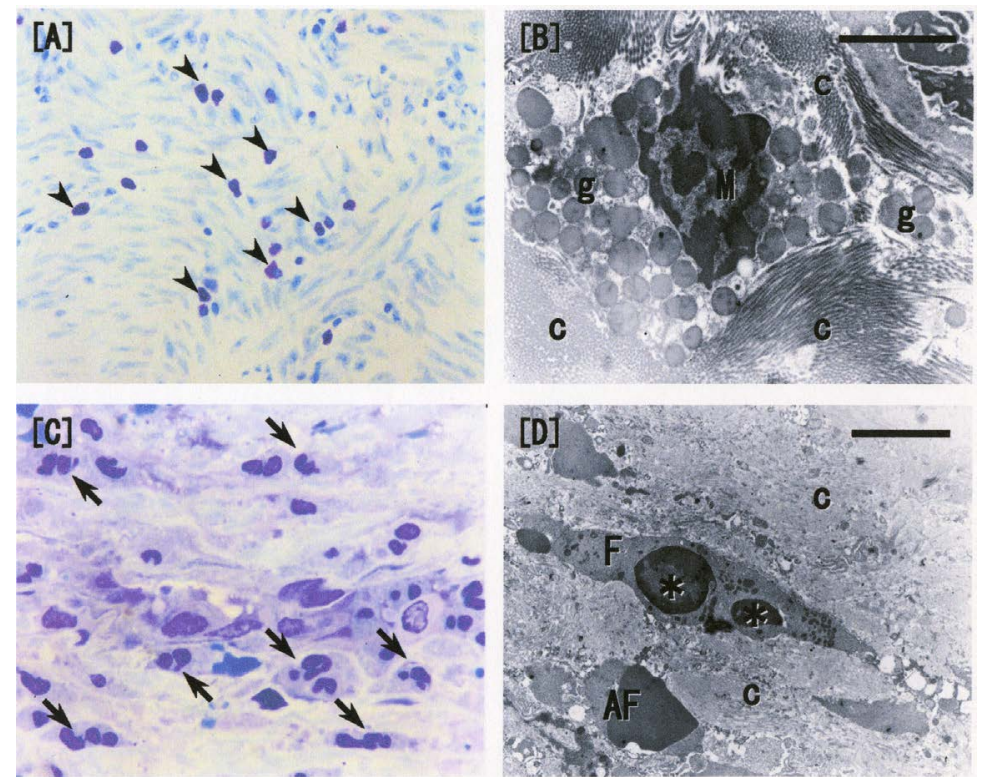

Figure 4. Light and electron microscopic examination of human endometriosis with and without LTR-A treatment. (A) Toluidine blue stain in a no-treatment case $(\times 200)$. Numerous mast cells (arrowhead: violet-stained cells) are observed; (B) Electron micrography in a no-treatment case. Degranulated mast cells (M, g: granules) and thick and dense collagen fibers (c) are observed. Bar scale, $3 \mu \mathrm{m}$; (C) Toluidine blue stain of LTR-A-treated cases $(\times 400)$. Mast cell is not observed at all, and numerous apoptotic cells (arrow) show nucleus fragments; (D) Electron micrography of an LTR-A-treated case. A fibroblast (F) shows nuclear fragments $(*)$, a typical apoptotic body of a fibroblast (AF) is also observed, and collagen fibers (c) became filamentous and thin. Bar scale, $3 \mu \mathrm{m}$. 
First, LTR-A-treated patients showed decreased infiltration and inhibition of degranulation (activation) by mast cells in the lesion. These findings indicate that the release of inflammatory mediators is inhibited by LTR-A treatment. As a result, some clinical symptoms (peritoneal pain, including dysmenorrheal pain) related to some mediators are relieved in LTR-A-treated patients. Recently, it has been reported that the presence of some of these mediators directly correlate with the degree of dysmenorrhea, which is one of the main symptoms of endometriosis [9] [10]. There is a distinct possibility that some clinical symptoms of endometriosis are relieved with the decrease of these mediators.

Furthermore, in LTR-A-treated cases, stromal proliferation in lesion areas showed frequent apoptosis of proliferating fibroblasts and degenerative changes in collagen fibers around these apoptotic fibroblasts. Specifically, the fibers were filamentous, thin, and partially condensed. These cells and fibers cause extensive adhesions, so these findings support the decrease in r-AFS score in LTR-A-treated cases and mirror the improved ability to laparoscopically lyse adhesions in LTR-A-treated patients, compared with no treatment. Actually, the adhesions of LTR-A-treated patients came off easily, and there was a little volume of blood loss in the laparoscope operation. It has the possibility that bleeding is restrained as the therapeutic effect so that leukotriene receptor antagonist may control functions of the leukotriene (blood vessel expansion and permeability progress), too.

Inasmuch as increases in inflammatory chemical mediators and extensive adhesions are recognized as infertility factors for endometriosis, anti-LT therapy must be efficacious not only in the treatment of clinical symptoms and lesions, but also in improving infertility in these patients [11]-[13]. Only the minimum number of patients that statistics processing was possible could obtain data by our investigation into the infertility. The investigation that has to continue in the long-term is difficult because the patients have stopped often seeing the doctor regularly when the clinical symptom is calmed down by anti-LT therapy.

Furthermore, since apoptosis, which represents physiological cell death rather than necrosis, was induced in proliferative fibroblasts, we feel that anti-LT therapy has minimal side effects. The endometriosis therapeutic-drug being made significance at present is hormone medicine, and many patients have to give up the therapy due to the strong side effect. Fortunately, no LTR-A-treated patient in our study discontinued therapy because of side effects, and some achieved pregnancy.

The presence of LTRs in endometriosis lesions is one reason why such therapeutic effects could be obtained. Generally, this receptor exists in lung smooth muscle cells, alveolar macrophages, mast cells, eosinophils and others. These cells are concerned with asthma, and LTR-As are used in asthma pharmacotherapy. The present study demonstrated for the first time that LTRs exist in endometriosis lesions, and suggests that LTR-As may be effective for endometriosis therapy.

Current medical treatment for human endometriosis is impacted by significant side effects, so patients frequently discontinue treatment, suffer a recurrence of lesions, and fail to achieve pregnancy. As current treatments also regulate the menstrual cycle, pregnancy is not possible while continuing treatment. In this study, all cells that expressed LTR in endometriosis lesions were influenced by anti-LT therapy.

\section{Conclusion}

Our results show that LTR-As have significant therapeutic effects, relieve some clinical symptoms, inhibit mast cell activation, and decrease stromal proliferation and adhesion formation. We are compiling additional data regarding the therapeutic effects of LTR-A on human endometriosis, and believe that anti-LT therapy offers a promising new therapeutic modality for human endometriosis.

\section{References}

[1] Houston, D.E., Noller, K.L., Melton III, L.J. and Selwyn, B.J. (1988) The Epidemiology of Pelvic Endometriosis. Clinical Obstetrics and Gynecology, 31, 787-800. http://dx.doi.org/10.1097/00003081-198812000-00005

[2] Dmownski, W.P., Braum, D. and Gebel, H.M. (1990) Endometriosis: Genetics and Immunologic Aspects. Current Concepts in Endometriosis. Alan R Liss, New York, 99-122.

[3] Sugamata, M., Ihara, T. and Uchiide, I. (2005) Increase of Activated Mast Cells in Human Endometriosis. American Journal of Reproductive Immunology, 53, 120-125. http://dx.doi.org/10.1111/j.1600-0897.2005.00254.X

[4] Uchiide, I., Ihara, T. and Sugamata, M. (2002) Pathological Evaluation of Rat Endometriosis Model. Fertility and Sterility, 78, 782-786. http://dx.doi.org/10.1016/S0015-0282(02)03327-7

[5] Ihara, T., Uchiide, I. and Sugamata, M. (2004) Light and Electron Microscopic Evaluation of Antileukotriene Therapy 
for Experimental Rat Endometriosis. Fertility and Sterility, 81, 819-823. http://dx.doi.org/10.1016/j.fertnstert.2003.08.029

[6] The American Fertility Society (1985) Revised American Fertility Society Classification of Endometriosis. Fertility and Sterility, 43, 351-352.

[7] Fawcett, D.W. and Jensh, R.P. (1997) Bloom \& Fawcett: Concise Histology. Chapman \& Hall, New York, 51-52.

[8] Ihara, T., Yamamoto, T., Sugamata, M., Okumura, H. and Ueno, Y. (1998) The Process of Ultrastructural Change from Nuclei to Apoptotic Body. Virchows Arch, 433, 443-447. http://dx.doi.org/10.1007/s004280050272

[9] Benebetto, C. (1989) Eicosanoids in Primary Dysmenorrheal, Endometriosis and Menstrual Migraine. Gynecological Endocrinology, 3, 71-94. http://dx.doi.org/10.3109/09513598909152454

[10] Yamaguchi, M. and Mori, N. (1990) Prostaglandin and Leukotriene Concentration of the Peritoneal Fluid of Endometriosis and Other Gynecologic Disorders in Secretory Phase. Prostaglandins, Leukotrienes and Essential Fatty Acids, 39, 43-45. http://dx.doi.org/10.1016/0952-3278(90)90170-P

[11] Karim, S.M. and Hillier, K. (1979) Prostaglandins in the Control of Animal and Human Reproduction. British Medical Bulletin, 35, 173-180.

[12] Fakih, H., Baggett, B., Holtz, G., Tsang, K.Y., Lee, J.C. and Williamson, H.O. (1987) Interleukin-1: A Possible Role in the Infertility Associated with Endometriosis. Fertility and Sterility, 47, 213-217.

[13] Ota, H., Igarashi, S., Hatazawa, J. and Taka, T. (1999) Immunohistochemical Assessmeny of Superoxide Dismutase Expression in the Endometrium in Endometriosis and Adenomyosis. Fertility and Sterility, 72, 129-134.

http://dx.doi.org/10.1016/S0015-0282(99)00152-1 